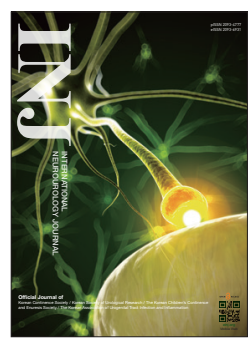

\title{
Time Course of Treatment for Primary Enuresis With Overactive Bladder
}

\author{
Young Jae Im¹, Jung Keun Lee ${ }^{1}$, Kwanjin Park ${ }^{1,2}$ \\ ${ }^{1}$ Department of Urology, Seoul National University College of Medicine, Seoul, Korea \\ ${ }^{2}$ Department of Urology, Seoul National University Children's Hospital, Seoul, Korea
}

\begin{abstract}
Purpose: To characterize the course of treatment for nonmonosymptomatic enuresis with overactive bladder $(\mathrm{OAB})$ in a real clinical setting.

Methods: Data from 111 OAB patients with moderate to severe enuresis were analyzed. The baseline analysis included a questionnaire, voiding diary, uroflowmetry with postvoid residual urine measurement, and plain abdominal radiography of the kidneys, ureters, and bladder (KUB). Following standard urotherapy for 1 month, anticholinergic medication was administered with or without laxatives. Desmopressin was added if there was a partial response to OAB. Patients were followed every 3 months to evaluate the status of $\mathrm{OAB}$ and enuresis. Multivariate analysis was performed to identify predictors associated with the lack of complete response (CR) in enuresis at 12 months.

Results: Following 12 months of treatment, $64 \%$ and $88 \%$ of patients experienced at least partial response in enuresis and $\mathrm{OAB}$, respectively. Urgency improved more quickly than enuresis, supporting the need to address daytime symptoms before enuresis. Seventy-nine patients (71\%) had fecal impaction on KUB and/or subjective constipation. The combination of anticholinergics with either laxatives or desmopressin fared better than anticholinergics alone. Daytime incontinence and anticholinergics-only treatment were associated with a lack of CR during 12 months of treatment.

Conclusions: The data confirmed the validity of addressing OAB before treating enuresis. The results of this study also highlight the need to address fecal impaction. Patients should be counseled about the need for a prolonged course of treatment before starting treatment. Anticholinergics should be accompanied with either desmopressin or laxatives for better control of enuresis.
\end{abstract}

Keywords: Constipation; Enuresis; Anticholinergics; Urinary bladder, Overactive

- Research Ethics: This study was performed according to the Helsinki Declaration (http://www.wma.net/en/30publications/10policies/b3/) and approved by the Institutional Review Board of Seoul National University Hospital (approval number: 1509-093-705).

- Conflict of Interest: No potential conflict of interest relevant to this article was reported.

\section{INTRODUCTION}

Nocturnal enuresis (NE) is a common voiding problem among children. Treatment is considered when NE persists in children over 5 years of age [1]. Several guidelines, including those of the International Children's Continence Society (ICCS), suggest that nonmonosymptomatic enuresis (NME) can be diagnosed when NE is associated with lower urinary tract dysfunction (LUTD) [2,3]. LUTD should be addressed prior to the treatment of enuresis per se. However, details regarding the evaluation and management of LUTD in patients with NME have been vaguely described. For example, such decisions depend on

Corresponding author: Kwanjin Park (D https://orcid.org/0000-0002-8926-3047 Department of Urology, Seoul National University Children's Hospital, 101 Daehak-ro, Jongno-gu, Seoul 03080, Korea

E-mail: urodori@naver.com, urodori9@snu.ac.kr / Tel: +82-2-2072-0695 / Fax: +82-2-742-4665

Submitted: January 9, 2018 / Accepted after revision: March 26, 2018 
the physician's discretion regarding the severity of LUTD that warrants treatment prior to addressing enuresis, the hierarchical order of instituting each treatment modality for LUTD, and the timing of ceasing LUTD treatment and starting enuresis management. The diagnosis and management of constipation for the treatment of LUTD or enuresis have likewise not been fully clarified. A simple question such as "Do you think your child has constipation?" may not be helpful for identifying the presence of constipation due to frequent inconsistencies among parental perceptions, symptoms, and the real amount of fecal loading [4,5]. Moreover, it is difficult to determine the severity of constipation that warrants treatment for LUTD. Given that adequate bowel decompression may take a long time, it is important to establish criteria for how much constipation should improve in order to address LUTD. Postponing treatment for enuresis until full decompression of the bowel may take several months to years, which may lead to reduced compliance and loss to follow-up.

Anticholinergics have been the mainstay for the treatment of overactive bladder $(\mathrm{OAB})[2,6]$. Since $\mathrm{OAB}$ is a common cause of LUTD in children, it is safe to say that anticholinergics are likely to play a major role in the treatment of NME. Although the use of anticholinergics is not recommended for the management of monosymptomatic enuresis (ME) [1], several studies have reported the successful use of anticholinergics to ameliorate or cure enuresis [7-10].

To understand the clinical course and results of NME treatment, we treated patients with NME as recommended by most guidelines [2,3], including standard urotherapy, constipation treatment (if present), and anticholinergics. The use of desmopressin was deferred until the occurrence of partial response (PR) of OAB. The analysis of our data is expected to provide helpful insights into the results of treatment when the principle of "addressing LUTD first" is strictly followed. Thus, the main purpose of our study was to characterize the course of treatment for NME with $\mathrm{OAB}$ in a real clinical setting.

This analysis may provide additional insights about which patients might show resistance to this treatment. The reason for treating LUTD prior to enuresis control is the perception that LUTD might be associated with a prolonged treatment period and resistance to treatment $[2,10,11]$. By understanding which patients may not respond to treatment, the treatment strategy could be tailored to obtain better results.

\section{MATERIALS AND METHODS}

With approval from the Institutional Review Board, and following human subject guidelines, we reviewed the medical records of 176 children aged 5-15 years who were diagnosed and treated with primary NE between July 2010 and July 2015. Twentyone patients who were diagnosed with ME were excluded from the review. Hence, data from 155 children who were treated for NME were eligible for review. None of these children had anatomical urinary tract abnormalities or neurological disorders. According to the ICCS definition, children who exhibited urgency or frequency or diurnal urgency incontinence were diagnosed with $\mathrm{OAB}$ [12].

All patients underwent a medical history and physical examination, completed a bladder diary, and were assessed using the modified Korean version of the Dysfunctional Voiding and Incontinence Symptom Score (DVISS) [13], the ROME III criteria for functional constipation [14], uroflowmetry, and postvoid residual urine measurement. Abnormal uroflow curves and elevated postvoid residual urine were seen in 23 patients. These patients were regarded to have other forms of voiding dysfunction, such as dysfunctional voiding. They were excluded from the review. Data regarding maximal and average voided volume and voiding frequency were extracted from bladder diaries. The average voided volume was determined after excluding the first morning voided volume. Expected bladder capacity was estimated following ICCS recommendations [12]. Simple plain radiography was performed routinely for the kidneys, ureters, and bladder before treatment to identify abnormalities in the spine and stool retention. Stool impaction was assessed based on the definitions presented by Marks et al. [4]. Rectal stool and total stool length (RSL and TSL, respectively) were measured as surrogate markers.

After the initial evaluation, standard urotherapy and treatment of constipation were first attempted. Standard urotherapy included demystification, education about urinary tract function, and advice regarding sound voiding habits, including lifestyle modifications such as timed voiding and adequate postvoid water consumption. In patients with a positive history of constipation according to the ROME III criteria, an RSL more than $6 \mathrm{~cm}$, or a TSL longer than $34 \mathrm{~cm}$, polyethylene glycol (10 g) or lactitol syrup $(15 \mathrm{~mL})$ was prescribed to decompress the bowel, depending on which was more palatable. Following a 1-month trial of standard urotherapy and/or laxatives, the further need to use anticholinergics was determined based on 
whether patients showed at least PR in daytime symptoms and complied with urotherapy. Treatment efficacy was measured in accordance with ICCS recommendations [12]. No response (NR) and PR were defined as reductions in enuresis of $<50 \%$ and 50\%-99\%, respectively. Complete response (CR) was considered to have occurred when there was 0-1 episode of enuresis during 3 months of follow-up. Patients who showed at least PR in urgency, were compliant on more than $80 \%$ of occasions based on their anamnesis, and were well-motivated continued nonpharmacological treatment. Anticholinergic treatment was reserved for those who did not meet those criteria. Based on the patient's ability to swallow tablets, a 5-mg tablet of solifenacin or $10 \mathrm{mg}$ of powdered propiverine was initially prescribed. All patients were followed regularly every 3 months. The use of desmopressin was deferred until they showed at least PR in control of urgency. At each follow-up, they were asked to provide information regarding the modified DVISS and a bladder diary.

We analyzed the demographic and clinical features of the patient population. Patients' response and disposition following initial nonpharmacological treatment were described. The clinical courses of urgency (as a surrogate marker of $\mathrm{OAB}$ ) and enuresis were plotted against the changing proportions of treatment responses every 3 months. The overall outcomes of $\mathrm{OAB}$ and enuresis following treatment were also assessed, and the features of patients who showed persistent NR at 12 months of follow-up were identified.

All categorical and continuous variables were reported as proportions and mean \pm standard error, respectively. The distribution of patients' responses was analyzed. Multivariate logistic regression analysis was performed to identify predictive factors for persistent NR. All statistical analyses were conducted using IBM SPSS Statistics ver. 21.0 (IBM Co., Armonk, NY, USA). Pvalues $<0.05$ were considered to indicate statistical significance.

\section{RESULTS}

\section{Patient Demographics and Clinical Features}

Initially, 132 patients were treated and followed. Twenty-one patients were lost within 3 months of follow-up. Their data were not included in this study. Hence, data from 111 patients who completed follow-up for 12 months were eligible for review. Of these patients, 54 (49\%) experienced CR at the 12-month follow-up visit. The baseline clinical characteristics of these 111 patients and a comparison of these variables between those who achieved CR and those who did not are shown in Table 1. The mean age of the patients was 6.7 years (range, 5-13 years). Approximately two-thirds of them were boys. All patients experienced enuresis for more than 3 days per week, while 75 (70\%) complained of almost daily enuresis. Urgency was found in all patients, and approximately $20 \%$ of

Table 1. Baseline demographics of 111 patients and comparison of clinical variables between patients who experienced complete resolution (CR) of enuresis and those who did not at 12 months of follow-up

\begin{tabular}{|c|c|c|c|c|}
\hline Parameter & $\operatorname{Total}(\mathrm{n}=111)$ & $\mathrm{CR}(+)(\mathrm{n}=54)$ & $\mathrm{CR}(-)(\mathrm{n}=57)$ & P-value \\
\hline Mean age (yr) & $6.8 \pm 1.8$ & $6.5 \pm 1.9$ & $7.1 \pm 2.9$ & 0.38 \\
\hline Male sex & $76(69)$ & 37 & 39 & 0.86 \\
\hline $\begin{array}{l}\text { No. of bed-wetting events } \\
\text { Moderate }(2-5 / \mathrm{wk}) \\
\text { Severe }(6-7 / \mathrm{wk})\end{array}$ & $\begin{array}{l}39(35) \\
72(65)\end{array}$ & $\begin{array}{l}23 \\
31\end{array}$ & $\begin{array}{l}16 \\
41\end{array}$ & 0.11 \\
\hline $\begin{array}{l}\text { DVISS questionnaires } \\
\text { Daytime incontinence } \\
\text { Urgency } \\
\text { Frequency ( } \geq 8 / \text { day) } \\
\text { Holding maneuver }\end{array}$ & $\begin{array}{c}22(20) \\
111(100) \\
47(42) \\
60(54)\end{array}$ & $\begin{array}{r}6 \\
54 \\
25 \\
28\end{array}$ & $\begin{array}{l}16 \\
57 \\
22 \\
32\end{array}$ & $\begin{array}{l}0.03 \\
1.00 \\
0.67 \\
0.21\end{array}$ \\
\hline $\begin{array}{l}\text { Bladder diary } \\
\text { MVV (mL) } \\
\text { AVV }(\mathrm{mL})\end{array}$ & $\begin{array}{l}176.4 \pm 68.5 \\
107.6 \pm 57.9\end{array}$ & $\begin{array}{r}163.2 \pm 72.4 \\
96.4 \pm 42.6\end{array}$ & $\begin{array}{r}162.6 \pm 59.8 \\
99.4 \pm 66.7\end{array}$ & $\begin{array}{l}0.62 \\
0.79\end{array}$ \\
\hline Constipation (ROME III $\geq 2$ ) & $41(37)$ & 16 & 25 & 0.12 \\
\hline Rectal stool length $\geq 6 \mathrm{~cm}$ & $74(67)$ & 33 & 41 & 0.23 \\
\hline Total stool length $\geq 34 \mathrm{~cm}$ & $65(59)$ & 29 & 36 & 0.31 \\
\hline
\end{tabular}

DVISS, Dysfunctional Voiding and Incontinence Symptom Score; MVV, maximal voided volume; AVV, average voided volume. 


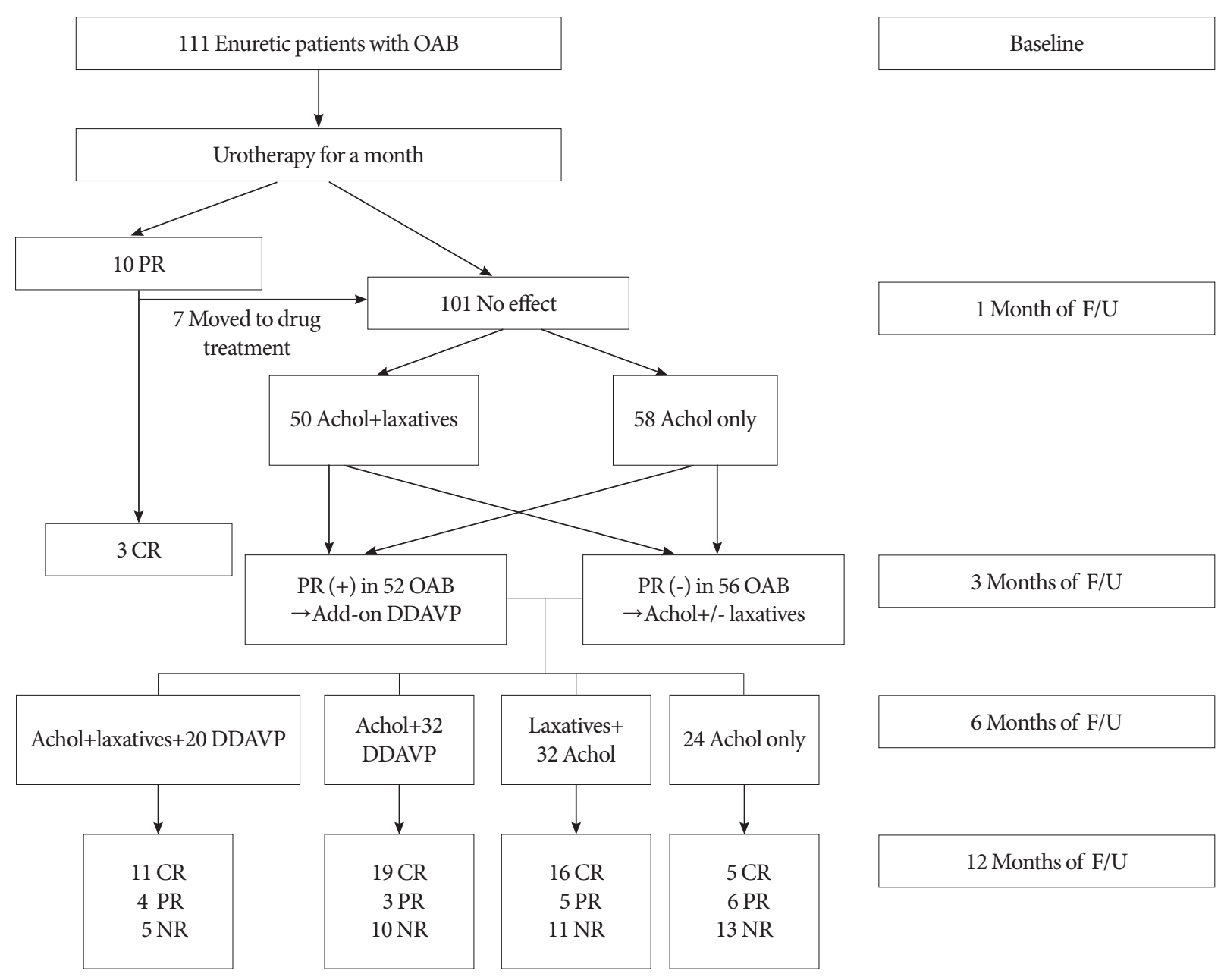

Fig. 1. Patient disposition and treatment outcomes during 12 months of follow-up. Achol, anticholinergics; F/U, follow-up; OAB, overactive bladder; DDAVP, desmopressin; CR, complete response; PR, partial response; NR, no response.

patients experienced daytime urinary incontinence. Bell- or tower-shaped uroflow patterns were seen in all cases. The mean maximal and averaged voided volumes in the bladder diaries were $176 \mathrm{~mL}$ and $108 \mathrm{~mL}$, respectively (corresponding to 68\% and $41 \%$ of age-adjusted volume, respectively). Seventy-nine patients were found to have either constipation or fecal impaction. The ROME III criteria for constipation were met in 41 patients (37\%). Fecal impaction (RSL more than $6 \mathrm{~cm}$ or TSL more than $34 \mathrm{~cm}$ ) was seen in 74 patients $(67 \%)$. Thirty-five patients (32\%) met the ROME III criteria for constipation and showed fecal impaction. Patients with daytime incontinence were significantly less likely to experience CR.

\section{Patient Disposition and Outcomes}

The initial treatment of urotherapy and constipation for 1 month led to PR in enuresis in 10 patients (9\%). Of them, 3 (30\%) eventually achieved CR within 3 months. The other 7 patients showed persistent enuresis and opted for pharmacological treatment. Hence, 108 patients received anticholinergics (Fig. 1).

The treatment of constipation was originally recommended for 79 patients with either subjective signs of constipation (ROME III) or fecal impaction (RSL more than $6 \mathrm{~cm}$ or TSL more than $34 \mathrm{~cm}$ ). However, only 53 patients $(67 \%)$ agreed to receive prolonged laxative treatment for fecal disimpaction. Along with 32 patients who did not need laxatives, the remaining 26 patients only received anticholinergics.

At 6 months of follow-up, according to changes in daytime symptoms, with urgency as the representative symptom, the need for additional desmopressin treatment was determined. A total of 4 treatment groups were identified. The overall treat- 


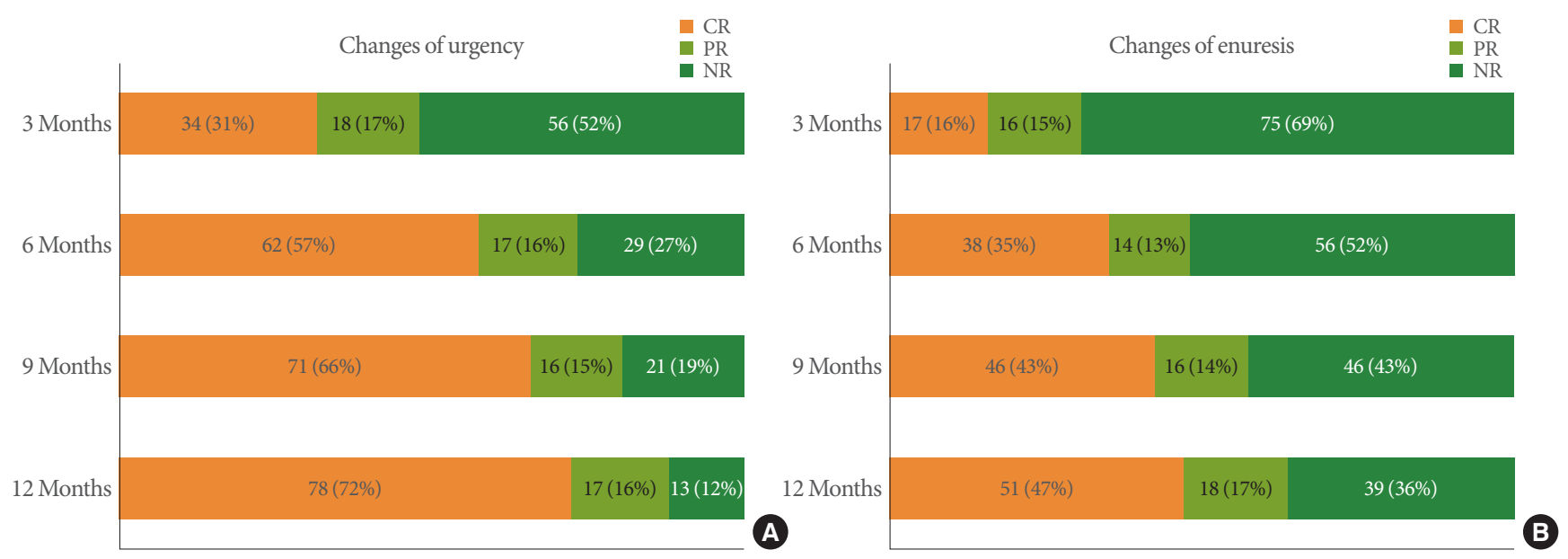

Fig. 2. Changes in the overall treatment responses for urgency (A) and enuresis (B) during 12 months of follow-up. CR, complete response; $\mathrm{PR}$, partial response; NR, no response.

Table 2. Multivariate logistic regression analysis of factors affecting the absence of complete response after 12 months of treatment

\begin{tabular}{lccc}
\hline Variable & Odds ratio & 95\% Confidence interval & P-value \\
\hline Severe enuresis & 0.46 & $0.11-1.88$ & 0.28 \\
Daytime incontinence & 5.96 & $2.11-17.20$ & 0.001 \\
Holding maneuver & 2.33 & $0.88-5.92$ & 0.08 \\
Constipation & 1.25 & $0.48-3.29$ & 0.64 \\
Rectal stool length $\geq 6 \mathrm{~cm}$ & 1.33 & $0.47-3.78$ & 0.58 \\
Total stool length $\geq 34 \mathrm{~cm}$ & 0.78 & $0.29-2.05$ & 0.62 \\
Treatment with only anticholinergics & 2.91 & $1.12-8.10$ & 0.04 \\
\hline
\end{tabular}

ment results for these 4 groups showed that CR and PR were achieved in 51 patients (47\%) and 18 patients (17\%), respectively. Those who only received anticholinergics showed the lowest rates of CR+PR (46\%) with respect to enuresis. A comparison with other groups revealed borderline significance $(\mathrm{P}=0.07$ by the chi-square test). Both anticholinergics and desmopressin were well tolerated in all patients.

To characterize the time course of the response to treatment, the changing distributions of clinical responses following medication in terms of urgency (A) and enuresis (B) at each followup are presented in Fig. 2.

To identify factors associated with failure to obtain CR in enuresis at 12 months of follow-up, forward stepwise multivariate logistic regression analysis was performed. The results showed that presentation with daytime incontinence and anticholinergics-only treatment were significantly associated with failure to achieve CR (Table 2).

\section{DISCUSSION}

In this study, we reviewed data regarding the treatment of enuretic patients with symptoms of OAB by standard urotherapy, constipation management, and subsequent anticholinergics. Following 12 months of treatment, $64 \%$ and $88 \%$ of patients experienced at least PR in enuresis and $\mathrm{OAB}$, respectively. To the best of our knowledge, this is the first study to present the detailed clinical course and long-term outcomes of NME patients with features of $\mathrm{OAB}$ in the real world after treatment according to currently used guidelines. The response rates for enuresis (corresponding to the proportion of patients with $\mathrm{CR}$ or $\mathrm{PR}$ ) were comparable to those of ME patients treated with desmopressin [15]. This suggests that NME with proper control of daytime symptoms could be successfully treated by desmopressin, with similar efficacy to the use of desmopressin for ME. The only notable difference was that the duration of treatment was longer in our cases than was the case for ME, as shown by 
the data of the Aarhus group [11]. This might be related to the need for time to stabilize the bladder prior to addressing enuresis. NME patients should receive counseling regarding this issue when considering such treatment. Without proper counseling, there may be problems in maintaining adequate compliance because some parents might take it for granted that enuresis is an easy-to-treat entity requiring only short-term pharmacological treatment.

Our results showed that anticholinergics alone were capable of controlling enuresis, as well as daytime symptoms, underscoring the role of bladder problems in the development of enuresis. Although the use of only anticholinergics was sufficient to control enuresis in some patients, more patients required combination therapy for enuresis to resolve. This supports the importance of other mechanisms that contribute to enuresis apart from bladder control. Since the optimal treatment for enuresis may vary according to patients' characteristics, further efforts should be made to elucidate the characteristics related to the efficacy of a given combination of treatments.

An interesting finding from the subgroup comparison was that the patients who received combination therapy with laxatives appeared to show better control of enuresis than those who used anticholinergics alone. This is consistent with a previous report showing a $63 \%$ reduction in enuresis following constipation treatment alone [16]. Moreover, this tendency could partially explain the low response rate in the anticholinergics-only treatment group, because the 19 patients who did not consent to constipation treatment were managed using only anticholinergics.

As in a previous trial of polyethylene glycol [17], the refusal of constipation treatment in 19 patients reflected difficulties in addressing constipation in patients with enuresis. This is related to the fact that no criteria have been generally accepted regarding the institution or discontinuation of constipation treatment in patients with LUTD. Moreover, it is currently unclear which tests are suitable for diagnosing constipation responsible for enuresis. Our data showed an unacceptable concordance rate between the subjective ROME III criteria and objective assessments of fecal impaction. Nonetheless, we tried to treat all patients who showed signs of fecal impaction, as well as those who had subjective constipation, based on the assumption that fecal impaction by itself could be problematic, regardless of the presence of symptoms.

The multivariate analysis revealed that presentation with daytime incontinence and anticholinergics-only treatment were associated with the lack of CR after 12 months of treatment. In- terpreting these results requires caution because these factors may not necessarily lead to eventual failure and refractoriness. Instead, the interpretation of these findings should be that more rigorous treatment of bladder and bowel problems is needed to accelerate the course of treatment and to achieve the resolution of enuresis. In this regard, the validity of the claim that combination treatment should be deferred until LUTD stabilizes may be questioned. However, this may result in the unnecessary treatment of those who show resolution of enuresis with anticholinergics alone.

The interpretation of our data requires careful scrutiny. First, it should be noted that the distinction of $\mathrm{OAB}$ from other voiding disorders was not based on uroflowmetry with simultaneous electromyography, which is the current standard [18]. Although only patients with bell/tower-shaped uroflow were selected, patients with other types of voiding disorders than $\mathrm{OAB}$ might have been included. Second, other treatment modalities, such as pelvic floor muscle exercise/biofeedback or an enuretic alarm, might be more effective for treating this condition. Applying different treatment modalities might be associated with different time courses in the improvement of enuresis and LUTD $[19,20]$. Third, this study was conducted in a tertiary care hospital. Therefore, inherent selection bias in patient recruitment may have been present. The retrospective nature of this analysis of real-world clinical data might have flaws. For example, the detailed treatment protocol may have varied across patients, even though the same treatment strategy was used. Finally, standard urotherapy was insufficiently applied to result in changes in enuresis despite its proven long-term efficacy in some patients [8]. However, the expectation of a prolonged time course and the modest efficacy inherent to standard urotherapy led us to avoid waiting for more than a month. In fact, 21 patients were lost within 3 months of treatment, implying that they were impatient for obtaining meaningful improvement of enuresis.

In conclusion, we acknowledge that a prolonged course of treatment is necessary to control NME and we confirm that $\mathrm{OAB}$ improves more quickly than enuresis. This supports the current guidelines that stress the need to treat daytime symptoms prior to treating enuresis per se. However, prolonged adherence to only anticholinergics to control daytime symptoms may lead to a protracted course of treatment. The proactive control of constipation and the early addition of desmopressin to anticholinergic therapy may facilitate the faster resolution of enuresis. 


\section{AUTHOR CONTRIBUTION STATEMENT}

- Full access to all the data in the study and takes responsibility for the integrity of the data and the accuracy of the data analysis: Kwanjin Park

- Study concept and design: Kwanjin Park

- Acquisition of data: Jun Keun Lee

- Analysis and interpretation of data: Young-Jae Im, Jung Keun Lee

- Drafting of the manuscript: Jun Keun Lee, Young-Jae Im

- Critical revision of the manuscript for important intellectual content: Young-Jae Im

- Statistical analysis: Kwanjin Park

- Obtained funding: Kwanjin Park

- Administrative, technical, or material support: Jun Keun Lee

-Study supervision: Jun Keun Lee

\section{REFERENCES}

1. Neveus T, Eggert P, Evans J, Macedo A, Rittig S, Tekgül S, et al. Evaluation of and treatment for monosymptomatic enuresis: a standardization document from the International Children's Continence Society. J Urol 2010;183:441-7.

2. Franco I, von Gontard A, De Gennaro M; International Childrens's Continence Society. Evaluation and treatment of nonmonosymptomatic nocturnal enuresis: a standardization document from the International Children's Continence Society. J Pediatr Urol 2013;9:234-43.

3. Vande Walle J, Rittig S, Bauer S, Eggert P, Marschall-Kehrel D, Tekgul S, et al. Practical consensus guidelines for the management of enuresis. Eur J Pediatr 2012;171:971-83.

4. Marks A, Vasquez E, Moylan S, Diaz-Saldano D, Schreiber J, Saker $\mathrm{M}$, et al. Definition of reliable, objective criteria by abdominal radiography to identify occult constipation in children with lower urinary tract symptoms. J Urol 2013;189:1519-23.

5. McGrath KH, Caldwell PH, Jones MP. The frequency of constipation in children with nocturnal enuresis: a comparison with parental reporting. J Paediatr Child Health 2008;44:19-27.

6. Franco I. Overactive bladder in children. Part 1: Pathophysiology. J Urol 2007;178(3 Pt 1):761-8.

7. Berkenwald A, Pires J, Ellsworth P. Evaluating use of higher dose oxybutynin in combination with desmopressin for refractory nocturnal enuresis. J Pediatr Urol 2016;12:220.e1-6.

8. Curran MJ, Kaefer M, Peters C, Logigian E, Bauer SB. The overactive bladder in childhood: long-term results with conservative management. J Urol 2000;163:574-7.
9. Hoebeke P, De Pooter J, De Caestecker K, Raes A, Dehoorne J, Van Laecke E, et al. Solifenacin for therapy resistant overactive bladder. J Urol 2009;182(4 Suppl):2040-4.

10. Vermandel A, de Wachter S, Wyndaele JJ. Refractory monosymptomatic nocturnal enuresis: a combined stepwise approach in childhood and follow-up into adolescence, with attention to the clinical value of normalizing bladder capacity. BJU Int 2005;96:629-33.

11. Rittig N, Hagstroem S, Mahler B, Kamperis K, Siggaard C, Mikkelsen MM, et al. Outcome of a standardized approach to childhood urinary symptoms-long-term follow-up of 720 patients. Neurourol Urodyn 2014;33:475-81.

12. Austin PF, Bauer SB, Bower W, Chase J, Franco I, Hoebeke P, et al. The standardization of terminology of lower urinary tract function in children and adolescents: update report from the Standardization Committee of the International Children's Continence Society. J Urol 2014;191:1863-5.e13.

13. Akbal C, Genc Y, Burgu B, Ozden E, Tekgul S. Dysfunctional voiding and incontinence scoring system: quantitative evaluation of incontinence symptoms in pediatric population. J Urol 2005;173:96973.

14. Rasquin A, Di Lorenzo C, Forbes D, Guiraldes E, Hyams JS, Staiano A, et al. Childhood functional gastrointestinal disorders: child/ adolescent. Gastroenterology 2006;130:1527-37.

15. Alloussi SH, Mürtz G, Lang C, Madersbacher H, Strugala G, Seibold J, et al. Desmopressin treatment regimens in monosymptomatic and nonmonosymptomatic enuresis: a review from a clinical perspective. J Pediatr Urol 2011;7:10-20.

16. Loening-Baucke V. Urinary incontinence and urinary tract infection and their resolution with treatment of chronic constipation of childhood. Pediatrics 1997;100(2 Pt 1):228-32.

17. Bush NC, Shah A, Barber T, Yang M, Bernstein I, Snodgrass W. Randomized, double-blind, placebo-controlled trial of polyethylene glycol (MiraLAX $\left.{ }^{\circledR}\right)$ for urinary urge symptoms. J Pediatr Urol 2013;9:597-604.

18. Van Batavia JP, Combs AJ, Fast AM, Glassberg KI. Use of non-invasive uroflowmetry with simultaneous electromyography to monitor patient response to treatment for lower urinary tract conditions. J Pediatr Urol 2014;10:532-7.

19. Ebiloglu T, Ergin G, Irkilata HC, Kibar Y. The biofeedback treatment for non-monosymptomatic enuresis nocturna. Neurourol Urodyn 2016;35:58-61.

20. Hoffmann A, Sampaio C, Nascimento AA, Veiga ML, Barroso U. Predictors of outcome in children and adolescents with overactive bladder treated with parasacral transcutaneous electrical nerve stimulation. J Pediatr Urol 2018;14:54.e1-54.e6. 